\title{
Comparing the effect of Topical Application of Breast Milk, Chlorhexidine and Dry Cord Care Methods on Bacterial Colonization in Umbilical Cord of Preterm Neonates in NICU: a randomized clinical trial
}

\author{
Mozhgan Yaghoobi ${ }^{1}$, Behjatossadat Bolandi ${ }^{2}$, Mohammadhasan Namaei $^{3}$, Gholamreza Faal ${ }^{4}$
}

\author{
${ }^{1}$ School of Medicine, Birjand University of Medical Sciences, Birjand, Iran \\ ${ }^{2}$ MD in Pediatric Nephrology, Assistant Professor, Department of Pediatrics, Faculty of Medicine, Birjand \\ University of Medical Sciences, Birjand, Iran \\ ${ }^{3} \mathrm{MD}, \mathrm{PhD}$, Professor of Microbiology, Birjand Infectious Diseases Research Center, Birjand University of Medical \\ Sciences, Birjand, Iran \\ ${ }^{4}$ MD in Neonatology, Assistant Professor, Department of Pediatrics, Faculty of Medicine, Birjand University of \\ Medical Sciences, Birjand, Iran
}

Type of article: Original

\begin{abstract}
Background: Preterm neonates have a higher tendency in developing infections and their umbilical cord serves as a good environment for bacterial growth.

Objective: The aim of this study was to compare the topical effects of breast milk, chlorhexidine and dry cord care methods on bacterial colonization of preterm neonates' umbilical cord in the Neonatal Intensive Care Unit (NICU)

Methods: The trial was one-centered, single-blind, and in phase 2 of clinical trials from Vali-e-Asr Educational Hospital, Birjand, Iran from January to June 2017. A sample of 75 preterm infants were recruited by convenience sampling method and assigned into one of the three groups of drying, chlorhexidine, and milk by using a table of random numbers ( $\mathrm{n}=25$ per group). Bacterial colonization and its density were assessed in all groups during the first 12 to 24 hours of admission, and again after 72 hours. A total of 25 subjects were entered in each group and the data were analyzed by Chi-square tests (Fisher's exact test), Kruskal-Wallis analysis, Wilcoxon test, McNemar's test, and ANOVA.

Results: The bacteria colonized in the newborns of groups I, II, and III before and after intervention were $64 \%$ and $36 \%(\mathrm{p}=0.03), 52$ and $20 \%(\mathrm{p}=0.008)$ and 64 and $32(\mathrm{p}=0.02)$ respectively. After the intervention, colonized bacteria were significantly decreased in all three groups.

Conclusion: Based on the results of this study, all three methods of drying approach, use of chlorhexidine, as well as breast milk were effective in controlling bacterial colonization in the umbilical cord of preterm neonates. Although there were no significant differences between the three groups, ultimately the use of these methods could be effective in reducing the need for antibiotics.

Trial registration: Prior to the study, the protocol of study was registered at Iran's Clinical Trials Registry (http://www.irct.ir) (Registration ID: IRCT2017090517756N27).

Funding: The present study was funded fully by Birjand University of Medical Sciences (grant number: 455078).

Keywords: Infant, Premature, Umbilical cord, Milk, Human, Chlorhexidine
\end{abstract}

Abbreviations / Acronyms:

CFU: Colony-Forming Unit; NICU: Neonatal Intensive Care Unit; PBS: Phosphate Buffered Saline; WHO: World Health Organization

\section{Corresponding author:}

Assistant Professor Dr. Gholamreza Faal, Department of Pediatrics, Faculty of Medicine, Birjand University of Medical Sciences, Birjand, Iran. Tel: +98.9153173566, Fax:+98.5132430076, Email: faalgh1@bums.ac.ir

Received: November 12, 2018, Accepted: April 10, 2019, Published: June 2019

iThenticate screening: April 06, 2019, English editing: May 12, 2019, Quality control: May 14, 2019

This article has been reviewed / commented by three experts

Funding / research project approval: Birjand University of Medical Sciences (Ref: 455078)

Ethics approval: Birjand University of Medical Sciences (Ref: Ir.bums.REC.1396.63)

(C) 2019 The Authors. This is an open access article under the terms of the Creative Commons Attribution-NonCommercialNoDerivs License, which permits use and distribution in any medium, provided the original work is properly cited, the use is non-commercial and no modifications or adaptations are made. 


\section{Introduction}

Preterm birth is one of the main causes of infant mortality. Preterm delivery complications in premature infants account for the greatest cause of direct death in infants and are responsible for $35 \%$ of the 3 million worldwide mortality rates in neonates (1). The umbilical cord is immediately necrotized after birth and provides a good environment for the growth of bacteria that causes infections in the neonate, especially in preterm neonates (2). Most factors that are colonized on the umbilical cord originate from the skin, the urethra and genital tract of the infant's mother, and most often include Staphylococcus aureus, Escherichia coli and Streptococcus $(3,4)$. Although the ratio of umbilical cord infection on infectious death in infants is still unclear, cord infection regardless of epithelial infection is known to be one of the main pathways for the introduction of bacteria into the infant's body (2). Since the umbilical cord has direct access to the bloodstream, bacterial colonization or infection can bring about several complications including intra-abdominal abscess, periumbilical cellulitis, thrombophlebitis in port or amyloid veins, peritonitis, and intestinal ischemia (5).

The World Health Organization (WHO) has recommended the use of a dry cord approach to cord storage since 1998 (6). The drying approach involves keeping the umbilical cord clean and exposing it to fresh air, or at most, storing it within a clean cloth. In centers where the health status is poorly maintained, or the infection rate is high, the WHO recommends using chlorhexidine $(5,7)$. There have been controversies regarding the effect of chlorhexidine usage on newborn mortality when born in hospitals. In fact, the use of topical chlorhexidine is a safe treatment approach, although there have been reports of small amounts of the substance existing in neonatal blood after topical use (8). Another traditional method with this regard is to recommend breast milk which offers antibacterial properties and causes an early removal of the cord $(9,10)$. Considering that there had not been a study until now that compares the effects of local breast milk, chlorhexidine and dry storage method specifically in preterm neonates admitted to Neonatal Intensive Care Unit (NICU), and all related studies have been with regard to term infants and outpatients, the aim of this study was to compare the topical effects of breast milk, chlorhexidine and dry cord care methods on bacterial colonization of preterm neonates' umbilical cord in the NICU.

\section{Material and Methods}

\subsection{Trial design}

The trial is one-centered, single-blind, and in phase 2 of clinical trials from Vali-e-Asr Educational Hospital, Birjand, Iran from January to June 2017. A sample of 75 preterm infants were recruited by convenience sampling method and assigned into one of the three groups of drying, chlorhexidine, and milk by using a table of random numbers ( $n=25$ per group). Data needed for this study were collected using a checklist containing treatment group, gender, delivery method, birth weight and bacterial density before and after treatment. The method for data collection was field-based with the use of interviews, examinations and laboratory tests. A trained nurse, who was blinded, collected the samples. Drug prescribing and drying was carried out by a qualified nurse in each work shift outside the research team. The researchers performed sampling as well as conducting microbiological tests.

\subsection{Selection criteria}

Inclusion criteria for this study were:

1) gestational age less than 37 weeks

2) need for admission in the neonatal intensive care unit

3) treatment with ampicillin and gentamicin antibiotics.

The following were set as the exclusion criteria:

1) dissatisfaction of the legal guardian(s) for participation

2) confirmed sepsis before bacterial culture

3) history of non-sterile childbirth

4) presence of anatomical malformation

5) history of perinatal asphyxia

6) presence of symptoms of omphalitis before the test

7) history of infections in the mother during pregnancy

8) prescribed antibiotics in the mother before delivery

9) prolonged rupture of fetal membranes

10) umbilical catheterization

11) any umbilical intervention during hospitalization; chorioamnionitis 


\subsection{Sample size}

The sample size required for this study was calculated using the following formula and based on a study by Taffazoli et al. (11) and with a confidence level of $95 \%$ and $\alpha=0.05$ and $\beta=0.2$ $\mathrm{n}=\mathrm{Z}_{1-\alpha}{ }^{2}[2 \mathrm{P}(1-\mathrm{P})]^{1 / 2}+\mathrm{Z}_{1-\beta}\left[\mathrm{P}_{1}\left(1-\mathrm{P}_{1}\right)+\mathrm{P}_{2}\left(1-\mathrm{P}_{2}\right)^{1 / 2}\right] /\left(\mathrm{P}_{1}-\mathrm{P}_{2}\right)^{2}$

\subsection{Randomization and blinding}

Infants were randomly assigned to one of three groups: 1, 2 and 3 (Figure 1). The study was single-blinded in that prescription or drying was performed by experienced nurses in various working shifts outside the research team. The researchers performed sampling as well as conducting microbiological tests.

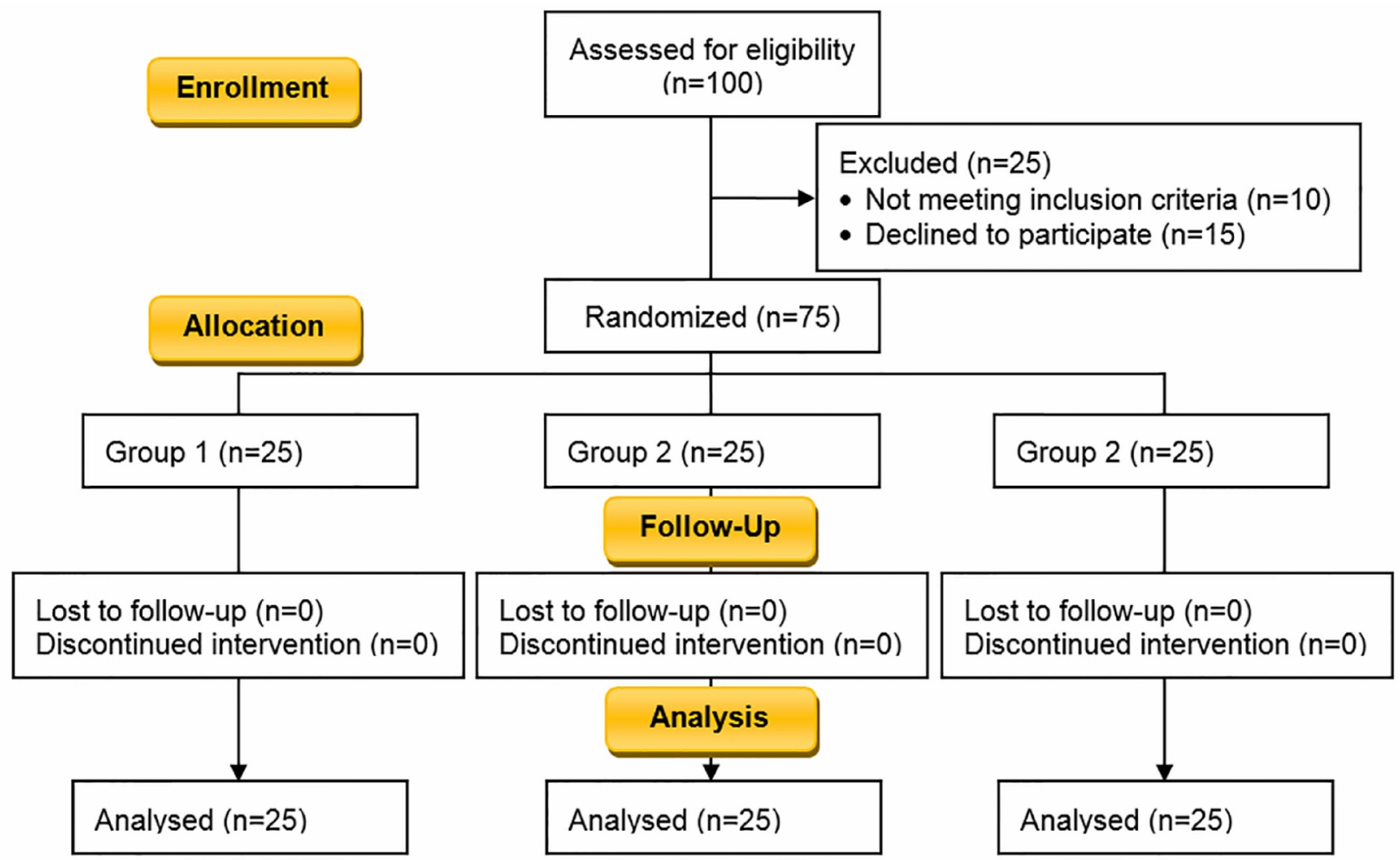

Figure 1. CONSORT Flow Diagram of the trial

\subsection{Intervention and measurements}

During the first 12 to 24 hours of admission, umbilical cord samples were collected from the neonates using a sterile swab; the wet sterilized swab was used to scrub the whole skin surface starting from the umbilical cord area with a radius of $1 \mathrm{~cm}$. Next, the specimen was prepared (taking into account the mean diameter of the umbilical cord, 1.5 $\mathrm{cm}$ plus 1 centimeter around it) and transferred to the laboratory in a Phosphate Buffered Saline (PBS) medium tube containing $5 \mathrm{cc}$ phosphate buffer saline with $\mathrm{pH} 7$. Serial dilutions were prepared in $0.1 \%$ of samples, then diluted 0.01 , and then the 0.01 dilutions were transferred onto an agar culture medium and Eosin methylene blue. Plates were stored at $37{ }^{\circ} \mathrm{C}$ for 48 hours. Next, specific numbers of colonies were determined by isolation from the bacterial strain of the grown bacteria in the media using standard laboratory method. Infants were randomly assigned to one of three groups. In each group, 12 intervention sessions were performed at six-hour intervals. In the dry group, the cord was kept dry only with sterile gas. In the chlorhexidine group, 2-3 cc based on the size of the umbilical cord, the umbilical cord, and 1 centimeter of the surrounding area were kept moist every six hours with $0.2 \%$ of chlorhexidine mouthwash, made by the Health Laboratory of the World Health Organization. In the breast milk group, between 2-3 cc from each infant's mother's milk based on the size of the umbilical cord, the entire umbilical cord and 1 centimeter of the surrounding area, were impregnated with milk every six hours. After 72 hours, with a minimum of six hours interval from the last intervention, samples were collected again from the umbilical cord surface swab and re-cultured. The results of two cultured groups were compared in each group followed by comparison in the two other groups. In order to evaluate the average bacterial density, the number of live bacteria per unit area of the body was used, which is calculated from the number of bacteria grown on the 
culture medium in the dilution image and divided by the sampling surface, which includes the total area of the scrubbed region minus the cord area average. The bacteria included different species of bacteria isolated from the sampling surface, which were identified by laboratory tests. In all groups, treatment began immediately after sampling was performed.

\subsection{Statistical analysis}

Data were analyzed using Statistical Package for the Social Sciences (SPSS) software version 21. Analytical analysis of qualitative data was performed by Chi-square test (Fisher's exact test) along with Kruskal-Wallis, Wilcoxon test, McNemar's test and ANOVA test. The significance level of $p$ was considered at a significance level of $\mathrm{p} \leq 0.05$.

\subsection{Research ethics}

All the procedures in this study were in conformity with the Declaration of Helsinki and were approved by the local Human Research Ethics Committee. Prior to the study, its protocol was approved by the Ethics Committee of Birjand University of Medical Sciences (Ir.bums.REC.1396.63) and registered at Iran's Clinical Trials Registry System (IRCT2017090517756N27).

\section{Results}

A total of 75 patients were enrolled in the study ( $n=25$ per group). Comparison of frequency distribution of variables (infants' age, birth weight, gestational age and birth method) in three groups of study showed no significant differences (Table 1). The frequency of colonized bacteria in the umbilical cord before and after intervention shown in Table 2 and Fisher's exact test indicates no difference between the groups with this regard. Table 2 also indicates no significant difference in the comparison of the type of bacteria colonized in the umbilical cord of preterm neonates after using drying method, chlorhexidine, and breast milk. The most common bacteria colonized in Group 1 and Group 3 were Staphylococcus Epidermidis (16\% in both groups) and for Group 2 was Staphylococcus Aureus $(12 \%)$.

Table 1. Comparison of frequency distribution of variables in three groups of study

\begin{tabular}{|l|l|l|l|l|l|}
\hline \multicolumn{2}{|l|}{ Variable } & Group 1 & Group 2 & Group 3 & Statistics \\
\hline Gender; n (\%) & Boy & $13(52)$ & $12(48)$ & $14(56)$ & $\mathrm{p}=0.85^{*}, \mathrm{X}^{2}=0.32$ \\
\cline { 2 - 5 } & Girl & $12(48)$ & $13(52)$ & $11(44)$ & \\
\hline \multirow{2}{*}{ Birth method; n (\%) } & Cesarean & $19(76)$ & $19(76)$ & $19(76)$ & $\mathrm{p}=1^{*}, \mathrm{X}^{2}=0.00$ \\
\cline { 2 - 6 } & Vaginal & $6(24)$ & $6(24)$ & $6(24)$ & \\
\hline Birth weight (g) & $1899 \pm 463.96$ & $1872.6 \pm 472.7$ & $1896.2 \pm 537.9$ & $\mathrm{p}=0.9^{* *}, \mathrm{~F}=0.02$ \\
\hline Gestational age (week) & $32.6 \pm 2.21$ & $32.32 \pm 2.34$ & $36.36 \pm 2.03$ & $\mathrm{p}=0.9^{* *}, \mathrm{~F}=0.2$ \\
\hline
\end{tabular}

Group 1: drying method; Group 2: chlorhexidine; Group 3: breast milk. * The result of Chi-squared test was not significant at the level of $\mathrm{p} \leq 0.05$. ** The ANOVA test was not significant at the level of $\mathrm{p} \leq 0.05$

Table 2. Frequency of colonized bacteria in umbilical cord before and after intervention

\begin{tabular}{|c|c|c|c|c|c|}
\hline \multicolumn{2}{|l|}{ Variables } & Group 1 & Group 2 & Group 3 & Statistics \\
\hline \multirow[t]{2}{*}{ Without growth; n (\%) } & Before & $9(36)$ & $12(48)$ & $9(36)$ & \multirow{12}{*}{$\begin{array}{l}\text { Before intervention: } \\
\text { Fisher exact }=7.2, \mathrm{p}=0.74 \\
\text { After intervention } \\
\text { Fisher exact }=7.2, \mathrm{p}=0.74\end{array}$} \\
\hline & After & $16(64)$ & $20(80)$ & $17(68)$ & \\
\hline \multirow[t]{2}{*}{ Staphylococcus epidermidis; n (\%) } & Before & $10(40)$ & $6(24)$ & $10(40)$ & \\
\hline & After & $4(16)$ & $0(0)$ & $4(16)$ & \\
\hline \multirow[t]{2}{*}{ Staphylococcus aureus; $\mathrm{n}(\%)$} & Before & $2(8)$ & $4(16)$ & $3(12)$ & \\
\hline & After & $3(12)$ & $3(12)$ & $1(4)$ & \\
\hline \multirow[t]{2}{*}{ Enterococcus; n (\%) } & Before & $2(8)$ & $1(4)$ & $0(0)$ & \\
\hline & After & $1(4)$ & $0(0)$ & $0(0)$ & \\
\hline \multirow[t]{2}{*}{ Escherichia coli; $\mathrm{n}(\%)$} & Before & $1(4)$ & $2(8)$ & $3(12)$ & \\
\hline & After & $1(4)$ & $2(8)$ & $3(12)$ & \\
\hline \multirow[t]{2}{*}{ Klebsiella; n (\%) } & Before & $1(4)$ & $0(0)$ & $0(0)$ & \\
\hline & After & $0(0)$ & $0(0)$ & $0(0)$ & \\
\hline
\end{tabular}

Group 1: drying method; Group 2: chlorhexidine; Group 3: breast milk.

Table 3 presents the mean of colonization of bacterial density before and after intervention as follows: Group 1

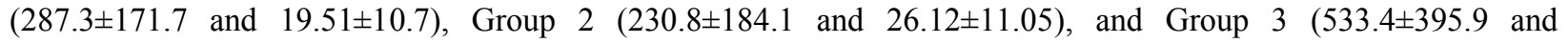


$32.04 \pm 18.03)$. A significant decrease was observable in all cases based on results of Wilcoxon test $(\mathrm{p}<0.001)$. Comparison of bacterial density changes in Table 3 by Kruskal-Wallis test indicated that there was no significant difference between the three groups $(p=0.4)$. Determination of the frequency of colonization of bacteria in the umbilical cord of all preterm neonates was first investigated and intervention was done, as presented in Table 3, there was no difference between the groups ( $\mathrm{p}=0.6$ and 0.4 , respectively). However, the growth of colonized bacteria in newborns in all three groups had a significant decrease. The number of colonized bacteria before and after the intervention on neonates in Group 1, Group 2, and Group 3 are as follows respectively: 64 and $36 \%$ ( $\mathrm{p}=0.03$ ), 52 and $20 \%(\mathrm{p}=0.008), 64$ and $32 \%(\mathrm{p}=0.02)$ and results of McNemar's test showed there are significance difference.

Table 3. Comparison of the average density of colonized bacteria $(\mathrm{CFU} / \mathrm{cm} 2)$ and growth of bacteria in the umbilical cord of preterm neonates before and after intervention

\begin{tabular}{|c|c|c|c|c|c|c|}
\hline \multirow[t]{2}{*}{ Groups } & \multicolumn{2}{|c|}{ Before intervention } & \multicolumn{2}{|c|}{ After intervention } & \multirow[t]{2}{*}{ Wilcoxon test } & \multirow{2}{*}{$\begin{array}{l}\text { Mean changes in bacterial } \\
\text { density }(\text { Mean } \pm \text { SD) }\end{array}$} \\
\hline & $\begin{array}{l}\text { Median } \\
\left(\mathrm{CFU} / \mathrm{cm}^{2}\right)^{* *}\end{array}$ & Mean \pm SD & Median & Mean \pm SD & & \\
\hline Group 1 & 118.47 & $287.3 \pm 171.7$ & 0 & $19.51 \pm 10.7$ & $\mathrm{p}<0.001, Z=-3.5$ & $-283.52 \pm 161.07$ \\
\hline Group 2 & 127.38 & $230.8 \pm 184.1$ & 0 & $26.12 \pm 11.05$ & $\mathrm{p}<0.001, \mathrm{Z}=-3.1$ & $-219.24 \pm 173.09$ \\
\hline Group 3 & 150.31 & $533.4 \pm 395.9$ & 0 & $32.04 \pm 18.03$ & $\mathrm{p}<0.001, \mathrm{Z}=-3.5$ & $-507.46 \pm 377.93$ \\
\hline Kruskal-Wallis Test* & \multicolumn{2}{|c|}{$\mathrm{X}^{2}=1.4, \mathrm{p}=0.4$} & \multicolumn{2}{|c|}{$\mathrm{X}^{2}=1.3, \mathrm{p}=0.5$} & - & $\mathrm{X}^{2}=1.5, \mathrm{p}=0.4$ \\
\hline \multicolumn{7}{|c|}{ Growth of bacteria $\left(\mathrm{CFU} / \mathrm{cm}^{2}\right)$ before and after intervention in three groups } \\
\hline & \multicolumn{3}{|c|}{ Group 1} & \multirow{2}{*}{\multicolumn{2}{|c|}{\begin{tabular}{|l|} 
Group 2 \\
$13\left(52^{\circ}\right.$
\end{tabular}}} & Group 3 \\
\hline Before intervention & \multicolumn{3}{|l|}{$16(64 \%)$} & \multirow{2}{*}{\multicolumn{2}{|c|}{\begin{tabular}{|l|l}
$13(52 \%)$ \\
$5(200$
\end{tabular}}} & $16(64 \%)$ \\
\hline After intervention & \multicolumn{3}{|l|}{$9(36 \%)$} & & $5(20 \%)$ & $8(32 \%)$ \\
\hline McNemar's test & \multicolumn{3}{|l|}{$\mathrm{p}=0.03$} & $\mathrm{p}=0.008$ & \multicolumn{2}{|l|}{$\mathrm{p}=0.02$} \\
\hline
\end{tabular}

CFU: Colony-Forming Unit; Group 1: drying method; Group 2: chlorhexidine; Group 3: breast milk.

$*$ The result of McNemar's test was not significant at $\mathrm{p} \leq 0.05 ; * *$ Colony-forming unit/square centimeter

\section{Discussion}

The WHO recommended clean dry cord care for infants born in health facilities and at home in 2013 (11). According to WHO recommendations, some factors such as topical antiseptic usage, infection and Cesarean section were responsible for delaying umbilical cord separation $(6,12)$. Most studies are conducted on well and term neonates, but our study was carried out on premature neonates who were admitted to the NICU. This is one of the most important differences in our study with other studies. This has made it difficult to compare with the results of other studies. In some studies, cord separation time has been identified as a useful criterion. However, it was decided that prevention of infection was the most important issue for premature infants, and for this reason we did not use the umbilical cord separation $(9,12,13)$. We did not find an article with an emphasis on the type of bacteria and the number of colonies in searches made in scientific publications such as PubMed. But in a systematic review with high-quality evidence from three community cRCTs, it is suggested that the use of $4 \%$ CHX reduces neonatal mortality in community settings, more so if this intervention is used in regions where infection rates are high, and with a moderate quality evidence suggesting that $4 \%$ CHX greatly reduces morbidity especially when started within 24 hours of birth in community settings and low-quality evidence, it is suggested that applying an antimicrobial is no better than leaving the cord to dry when in a high-income or middle income hospital setting (14).

Our study focuses on the type of germs, not on prognosis. It was revealed to us that there was no statistically significant difference between groups in terms of the type of microbial growth that developed. the results of the present study stated that the most common bacteria colonized in Group 1 and Group 3 was Staphylococcus epidermidis (which are non-pathogenic and natural flora of skin), and for Group 2 was Staphylococcus aureus (which is a pathogen bacteria). In a clinical trial study by Abbaszadeh et al., a significant correlation was not found between signs of infection (discharge, redness and swelling) in both groups. No cases of granuloma formation and sepsis were found and none of the neonates needed to be hospitalized. In other words, they found that mother's milk could be as effective as chlorhexidine (broad-spectrum antibiotics) in reducing signs of infection of cord. This result is matched with a study by Ahmadpour-Kacho et al. Regarding the fact that redness and swelling can be a sign of the growth of microbes on the umbilical cord, it can be compared with our study, in which we also did not make a significant difference between the rate of microbial growth in both breast milk and chlorhexidine groups $(9,13)$. The study of Vural and Kisa, which compared cord care using topical breast milk, drying, and povidone-iodine on 150 healthy, full-term newborns could not find significant differences between the three groups in terms of omphalitis 
occurrence. They suggested the cultural practice of applying human milk to the umbilical cord stump appears to have no adverse effects. Because omphalitis is associated with microbial infection, the findings of the study are consistent with our findings (10). Taffazoli et al. found the most common cultured organisms were S. epidermidis, S. aureus, E. coli and Klebsiella pneumoniae in the umbilical stump, there were significant differences between two groups in colonization rate (dry cord method vs breast milk method). We found the most common organisms are Staphylococcus epidermidis, Staphylococcus epidermidis, Enterococcus, Escherichia coli, and Klebsiella but we could not find significant differences between the three groups in colonization rate (dry cord method vs. breast milk method vs. chlorhexidine method) (15). The studies of Mahrous et al. and Taffazoli et al. are of a few studies that have shown that other methods aside from maintaining dryness and preservation method in sterile delivery conditions have indeed a significant effect on the reduction of pathogenic bacterial level $(15,16)$. Nevertheless, it should be noted that cord storage strategies, except for the use of chlorhexidine, have not been systematically evaluated so far, although it has been shown that the use of traditional methods such as local use of ash, breast milk, or herbal teas only provide a suitable environment for the growth of bacteria and have no clinical benefit (2). However, it is possible that using breast milk in the umbilical cord could lead to a decrease in the removal duration of the umbilical cord, and consequently lead to decreased chances of infection and bacterial colonization $(8,10)$.

Various therapeutic approaches other than the use of breast milk have been evaluated in terms of umbilical cord care. In a study by Evens et al. where umbilical cord care in premature newborns in the time of separation as well as bacterial colonization was investigated, neonates were randomly treated with alcohol or with dryness (17). Ozdemir et al., who evaluated the effect of different antiseptics on cord separation time, also showed that chlorhexidine application was the most effective agent in decreasing colonization, as it significantly increased cord separation time (18).

Finally, the results of the present study showed that there were no statistically significant differences between the average density and type of bacteria colonized in the umbilical cord of the three groups after the intervention. Of course, it should be noted that each of the methods of drying, use of chlorhexidine, as well as the use of breast milk were effective in controlling the bacterial colonization of the umbilical cord of the newborns. The significance of the present study was the evaluation of three main different methods for umbilical cord care in a sterile hospital condition, with a single-blind approach, which offers a uniqueness compared to other studies in the field. The limitations of this study however, would be the lack of evaluation of the effect of each method on the time of umbilical cord separation. The present study was conducted on preterm neonates born in sterile conditions and the results should not be generalized and applied to infants born at home.

\section{Conclusions}

The results of the present study indicate that after the intervention, colonized bacteria were significantly decreased in all three cord care groups (breast milk method, dry method and chlorhexidine method). The amount of reduction was not different between the groups. Based on the results of this study, all three methods of drying approach, use of chlorhexidine, as well as breast milk were effective in controlling bacterial colonization in the umbilical cord of preterm neonates. Although there were no significant differences between the three groups, ultimately, the use of these methods could be effective in reducing the need for antibiotics.

\section{Acknowledgments:}

This paper is the result of student thesis No. 961 at Birjand University of Medical Sciences. The authors would like to thank the research deputy of Birjand University of Medical Sciences, the kind employees of the NICU department of Vali-e-Asr Hospital in Birjand who have offered the best cooperation to this research, and everyone who sincerely participated in this research.

\section{Trial registration:}

The protocol of study was registered at Iran's Clinical Trials Registry System (http://www.irct.ir) (IRCT2017090517756N27).

\section{Funding:}

The present study was funded fully by Birjand University of Medical Sciences (grant number: 455078).

\section{Conflict of Interest:}

There is no conflict of interest to be declared. 


\section{Authors' contributions:}

GF designed the study and performed the data analysis. All authors contributed in data acquisition. All authors contributed in drafting the manuscript and GF revised the manuscript. All authors are accountable for all aspects of the work

\section{References:}

1) Liu L, Johnson HL, Cousens S, Perin J, Scott S, Lawn JE, et al. Global, regional, and national causes of child mortality: an updated systematic analysis for 2010 with time trends since 2000. The Lancet. 2012; 379(9832): 2151-61. doi: 10.1016/S0140-6736(12)60560-1. PMID: 22579125.

2) Stewart D, Benitz W. Umbilical cord care in the newborn infant. Pediatrics. 2016; 138(3): e20162149. doi: 10.1542/peds.2016-2149. PMID: 27573092.

3) Camacho-Gonzalez A, Spearman PW, Stoll BJ. Neonatal infectious diseases: evaluation of neonatal sepsis. Pediatr Clin North Am. 2013; 60(2): 367-89. doi: 10.1016/j.pcl.2012.12.003. PMID: 23481106, PMCID: PMC4405627.

4) Cortese F, Scicchitano P, Gesualdo M, Filaninno A, De Giorgi E, Schettini F, et al. Early and late infections in newborns: where do we stand? A review. Pediatr Neonatol. 2016; 57(4): 265-73. doi: 10.1016/j.pedneo.2015.09.007. PMID: 26750406.

5) Samuel M, Freeman NV, Vaishnav A, Sajwany MJ, Nayar MP. Necrotizing fasciitis: a serious complication of omphalitis in neonates. Journal of pediatric surgery. 1994; 29(11): 1414-6. doi: 10.1016/0022-3468(94)90133-3.

6) Organization WH. Post partum care of the mother and newborn, care of the umblical cord; 1998.

7) Organization $\mathrm{WH}$. WHO recommendations on postnatal care of the mother and newborn: World Health Organization; 2014.

8) Sinha A, Sazawal S, Pradhan A, Ramji S, Opiyo N. Chlorhexidine skin or cord care for prevention of mortality and infections in neonates. Cochrane Database Syst Rev. 2015; (3): CD007835. doi: 10.1002/14651858.CD007835.pub2. PMID: 25739381.

9) Abbaszadeh F, Hajizadeh Z, Jahangiri M. Comparing the impact of topical application of human milk and chlorhexidine on cord separation time in newborns. Pakistan journal of medical sciences. 2016; 32(1): 239. doi: 10.12669/pjms.321.8223. PMID: 27022383, PMCID: PMC4795877.

10) Vural G, Kisa S. Umbilical Cord Care: A Pilot Study Comparing Topical Human Milk, Povidone - Iodine, and Dry Care. J Obstet Gynecol Neonatal Nurs. 2006; 35(1): 123-8. doi: 10.1111/j.15526909.2006.00012.x. PMID: 16466360 PMCID: PMC2364713.

11) Quattrin R, Iacobucci K, De Tina AL, Gallina L, Pittini C, Brusaferro S. 70\% Alcohol Versus Dry Cord Care in the Umbilical Cord Care: A Case-Control Study in Italy. Medicine (Baltimore). 2016; 95(14): e3207. doi: 10.1097/MD.0000000000003207. PMID: 27057849, PMCID: PMC4998765.

12) Golshan M, Hossein N. Impact of ethanol, dry care and human milk on the time for umbilical cord separation. J Pak Med Assoc. 2013; 63(9): 1117-9.

13) Ahmadpour-Kacho M, Zahedpasha Y, Hajian K, Javadi G, Talebian H. The effect of topical application of human milk, ethyl alcohol $96 \%$, and silver sulfadiazine on umbilical cord separation time in newborn infants. Arch Iran Med. 2006; 9(1): 33-8.

14) Karumbi J, Mulaku M, Aluvaala J, English M, Opiyo N. Topical umbilical cord care for prevention of infection and neonatal mortality. Pediatr Infect Dis J. 2013; 32(1): 78-83. doi: 10.1097/INF.0b013e3182783dc3. PMID: 23076382, PMCID: PMC3785148.

15) Taffazoli M, Farahani L, Mohammadzadeh A, Esmaeeli H, Ghazvini K. Dose topical application of breast milk affect on bacterial colonization in umbilical cord? Koomesh. 2008; 10(1).

16) Mahrous ES, Darwish MM, Dabash SA, Ibrahim M, Abdelwahab SF. Topical application of human milk reduces umbilical cord separation time and bacterial colonization compared to ethanol in newborns. Translational Biomedicine. 2012; 3(1).

17) Evens K, George J, Angst D, Schweig L. Does umbilical cord care in preterm infants influence cord bacterial colonization or detachment? J perinatol. 2004; 24(2): 100-4. doi: 10.1038/sj.jp.7211027. PMID: 14762447.

18) Ozdemir H, Bilgen H, Topuzoglu A, Coskun S, Soyletir G, Bakir M, et al. Impact of different antiseptics on umbilical cord colonization and cord separation time. The Journal of Infection in Developing Countries. 2017; 11(02): 152-7. doi: 10.3855/jidc.7224. PMID: 28248676. 